\title{
Prospectively Predicting Adult Depressive Symptoms from Adolescent Peer Dysfunction: A
} Sibling Comparison Study

Carter J. Funkhouser, ${ }^{\mathrm{a}, \mathrm{b}}$ Sameer A. Ashaie, ${ }^{\mathrm{c}}$ Marc J. Gameroff ${ }^{\mathrm{d}, \mathrm{e}}$, Ardesheer Talati ${ }^{\mathrm{d}, \mathrm{e}}$, Jonathan Posner $^{\mathrm{d}, \mathrm{e}}$, Myrna M. Weissman, ${ }^{\mathrm{d}, \mathrm{e}}$ \& Stewart A. Shankman ${ }^{\mathrm{a}}$

aNorthwestern University Department of Psychiatry and Behavioral Sciences 680 N. Lake Shore Drive, Chicago, IL 60611

${ }^{b}$ University of Illinois at Chicago

Department of Psychology

1007 W. Harrison Street, Chicago, IL 60607

'Shirley Ryan AbilityLab

Center for Aphasia Research and Treatment

355 E. Erie Street, Chicago, IL 60611

${ }^{\mathrm{d} C o l u m b i a}$ University, Vagelos College of Physicians and Surgeons

Department of Psychiatry

1051 Riverside Drive, New York, NY 10032

eNew York State Psychiatric Institute

Division of Translational Epidemiology

1051 Riverside Drive, New York, NY 10032 


\begin{abstract}
Previous studies have shown that peer dysfunction in adolescence predicts depression in adulthood, even when controlling for certain individual- and/or family-level characteristics. However, these studies have not controlled for numerous potential familial confounders, precluding causal inferences. The present study therefore used a sibling comparison design (i.e., comparing siblings within families) to test whether peer dysfunction (e.g., lack of friendships, victimization) in adolescence continues to predict depression in adulthood after accounting for unmeasured familial confounds and individual characteristics in adolescence. Participants'
\end{abstract} $(N=85)$ dysfunction with peers was assessed in adolescence $\left(M_{\mathrm{age}}=13.21, S D=3.47\right)$ by self- and parent-report, and adult depressive symptoms was assessed up to five times, up to 38 years later. Multilevel modeling was used to examine the effect of adolescent peer dysfunction on adult depressive symptoms after adjusting for familial confounds and/or individual characteristics in adolescence (e.g., baseline depressive symptoms, dysfunctional relations with siblings/parents). Both self-reported $(b=1.28, p<.001)$ and parent-reported $(b=0.56, p=.032)$ adolescent peer dysfunction were associated with greater depressive symptoms severity in adulthood in unadjusted models. Self-reported (but not parent-reported) adolescent peer dysfunction continued to predict adult depressive symptoms after controlling for familial confounding and measured covariates such as adolescent depressive symptoms and relations with siblings and parents $(b=1.06, p=.035)$. Although confidence intervals were wide and the potentially confounding effects of numerous individual-level factors were not ruled out, these findings provide preliminary evidence that perceived peer dysfunction in adolescence may be an unconfounded risk factor for depressive symptoms in adulthood.

Keywords: peer relationships, social factors, relationships, risk factors, depression 
Depression is a leading cause of disability (Friedrich, 2017) and has a peak onset beginning in adolescence (Avenevoli et al., 2015), a developmental period characterized by change and increased autonomy. Identifying depression risk factors in adolescence and mechanisms underlying their association with depression later in life is therefore critical for reducing depression's public health burden through prevention. Peer functioning and relationships are particularly important sources of support in adolescence (Larson et al., 1996). Numerous etiological theories of depression emphasize the role of peer stressors and lack of peer support in the development or exacerbation of adolescent depression (Cohen \& Wills, 1985; Hammen, 2005; Panzarella et al., 2006), and peer dysfunction (e.g., peer victimization, lack of friendships, affiliation with deviant peers) is strongly associated with depression and other psychopathologies in adolescence (e.g., Roach, 2018). Importantly, several longitudinal studies have found that peer dysfunction in adolescence predicted depression later in adolescence (Michelini et al., 2021; Reijntjes et al., 2010) and in early and middle adulthood (Bagwell et al., 2001; Bean et al., 2019; Copeland et al., 2013; Landstedt et al., 2015; Modin et al., 2011), suggesting that adolescent peer dysfunction may be a valid prospective indicator of depression risk. Importantly, some other studies have found support for a symptoms-driven model (i.e., depressive symptoms -> peer dysfunction; Kochel et al., 2012; Shapero et al., 2013) and it is plausible that peer dysfunction and depressive symptoms reciprocally reinforce each other (Rudolph, 2009). If so, this transactional process may contribute to the maintenance or recurrence of depressive symptoms.

Although the association between adolescent peer dysfunction and adult depression has been consistently observed across studies, it is important to rule out the possibility that this relationship is due to confounders that cause both adolescent peer dysfunction and adult 
depression. Determining whether this association is confounded (i.e., spurious) is essential for developing preventative interventions based on causal mechanisms underlying risk and determining which adolescents will benefit from preventative interventions (Cuijpers et al., 2012). For example, if the association is entirely due to a confounder, a preventative intervention that successfully targets adolescents' peer functioning would have no impact on depression risk in adulthood. Previous studies indicate that statistically controlling for certain individual-level (e.g., sex, baseline depression severity) and/or family-level (e.g., socioeconomic status) confounders attenuated - but did not fully explain - this relationship (Bean et al., 2019; Bowes et al., 2015; Landstedt et al., 2015). However, many potential family-level confounders (e.g., parental depression, maladaptive home environment, genetic characteristics; Gjerde et al., 2017; Silberg et al., 2010) have not been considered in prior studies, precluding stronger "causal" inferences. For example, maladaptive parental interpersonal behaviors, cognitions, and affective processes may be passed from parent to offspring via social learning and subsequently contribute to peer dysfunction (e.g., Goodman et al., 1993). Maladaptive parental behaviors (e.g., negative parenting) may also function as stressors that directly contribute to offspring depression (Goodman \& Gotlib, 1999; Hammen et al., 2004) and later increases in depressive symptoms across adolescence (Garber \& Cole, 2010).

Although randomized experiments are ideal for eliminating potential confounding effects, quasi-experimental designs such as the sibling comparison design can rule out certain confounders in cases when randomization is infeasible. The sibling comparison design compares siblings from the same family who are discordant on an 'exposure' (e.g., peer dysfunction), and can be thought of as a matched case-control study (Frisell, 2020). Statistically comparing siblings eliminates the effect of all unmeasured environmental (e.g., race/ethnicity, 
socioeconomic status, neighborhood factors, family characteristics) and genetic confounders shared between siblings (Lahey \& D'Onofrio, 2010). Importantly, the sibling comparison design's strength of controlling for all familial confounders without measuring them comes with the tradeoff that it is difficult to know which specific genetic and environmental confounders are shared between siblings (and thus controlled for) in a particular study. Thus, sibling comparison analyses represent a useful first step to test - and potentially rule out - a large set of potential confounders that would be exceedingly difficult to measure and control for in a sample of unrelated individuals. If a sibling comparison analysis suggests familial confounding, further studies would then be necessary to identify the specific confounding factors.

Although there is limited research on this topic comparing biologically related individuals, twin studies (which similarly compare siblings [i.e., twins]) of 6- to 10-year-olds suggest that peer difficulties and bullying victimization in childhood are primarily attributable to genetic characteristics (Ball et al., 2008; Boivin et al., 2013; Brendgen et al., 2017; MorneauVaillancourt et al., 2019). Additionally, a retrospective study of female twins reported that the association between peer victimization in adolescence and depressive episodes in adulthood was $60 \%$ "causal" (i.e., unexplained by genetic or environmental confounds) and $40 \%$ attributable to genetic factors that influence both adolescent peer victimization and adult depression (Kretschmer et al., 2018). This study did not consider other types of peer dysfunction, however, and its retrospective assessment of peer victimization 40 years earlier and focus on females may have limited the validity and generalizability of these results. In sum, very few studies have used sibling designs to control for familial confounding, and those that have (a) focused on peer victimization without considering other forms of peer dysfunction, (b) measured peer 
dysfunction in childhood rather than adolescence, and/or (c) had notable methodological limitations (e.g., retrospective assessment).

The present study used a prospective design to (1) replicate the previously observed relationship between peer dysfunction in adolescence and depressive symptoms in adulthood using a follow-up of up to 38 years, and (2) test whether this relationship remained after accounting for unmeasured genetic or environmental confounds shared between siblings and/or measured covariates that vary between siblings. Notably, dysfunctional relations with parents and siblings during adolescence have also predicted depression in adulthood (Reinherz et al., 2003; Waldinger et al., 2007), and thus were included as covariates to test whether the association between adolescent peer dysfunction and adult depressive symptoms was independent of these other interpersonal domains. We also conducted supplementary analyses estimating the unique effects of self- versus parent-reported adolescent peer dysfunction to test specificity.

\section{Methods}

\section{Participants}

This study used data from a longitudinal, three-generation cohort study. The initial sample (G1s) consisted of white, non-Hispanic, predominantly middle class adults with either Major Depressive Disorder (MDD) or no history of psychiatric illness or treatment recruited from outpatient psychiatric clinics or the community in the early 1980s. G1s, their children (G2s), and their grandchildren (G3s) completed numerous waves of assessments over the following decades. The present study focused on G2s because G1s were not assessed in adolescence and G3s had not yet reached middle age. G2s first participated at wave 1 or 2 (approximately 1982-1984), and later completed assessments of depressive symptoms at five 
follow-up waves in adulthood (ending in 2020). There were 126 G2s with non-missing wave 1 self- or parent-report peer dysfunction data, and 85 participants available for analysis after excluding those without any depression assessments in adulthood. ${ }^{1}$ The sample consisted of 15 singletons and 70 participants with at least one participating biological sibling (nested within 34 families).

The average age at baseline was $13.21(S D=3.47)$, and participants were followed up for an average of 30.86 years $(S D=7.16$; range=17.1-37.8). The five follow-ups in adulthood were spaced approximately five years apart and participants completed an average of $2.62(S D=1.43)$ follow-ups. The average ages at the first and last follow-ups were $30.86(S D=3.63)$ and 49.03 $(S D=4.11)$, respectively. More detailed information about the age at each follow-up and demographic and clinical characteristics are presented in Table 1. Study procedures were approved by the institutional review board. Written informed consent was obtained from adults for themselves and minors, and verbal assent was obtained from minors.

\section{Measures}

\section{Social Adjustment Inventory for Children and Adolescents}

The Social Adjustment Inventory for Children and Adolescents (SAICA; John et al., 1987) is a semi-structured interview delivered separately to parent and youth, and assesses adolescents' functioning in many domains. The present study focused on three subscales measuring interpersonal functioning with peers, siblings, and parents, respectively. Each subscale contains items assessing either adaptive functioning or problems in that interpersonal domain. Items are rated on a 4-point Likert scale ranging from 'very true' to 'not at all true' (for items regarding adaptive functioning) or 'not a problem' to 'severe problem' (for items regarding

\footnotetext{
${ }^{1}$ Three G2 participants who first participated at wave 2 were included to maximize power, and their wave 2 data was used as baseline.
} 
problems). The 16-item peer functioning subscale contains items assessing various components of adaptive peer functioning or problems with peers. Assessed components of adaptive peer functioning include acceptance (e.g., "makes new friends easily", "has a steady group of friends"), popularity (e.g., "is popular with others"), close friendships ("has one or two special friends"), and leadership (e.g., "is a leader"). Assessed problems with peers include victimization ("is teased/bullied by other kids"), bullying perpetration ("bullies other kids"), shyness ("is shy with other kids"), difficulty maintaining friendships (e.g., "has trouble keeping friends"), and deviant peer affiliation (e.g., "hangs out with other kids who get into trouble"). ${ }^{2}$

Dysfunction in sibling and parent relations was measured using the 9-item sibling relations and 10 -item parent relations subscales, respectively. ${ }^{3}$ The sibling relations subscale contains three items assessing positive interactions with siblings ("plays or does things with them", "is friendly toward/affectionate with them", "talks with them") and six items assessing problems with siblings such as avoidance ("avoids contact with siblings", "is avoided by siblings"), bullying (“scapegoats/bullies siblings", "is scapegoated/bullied by siblings”), and physical aggression ("injures siblings", "is injured by siblings"). The parent relations subscale separately assesses positive interactions with one's mother and father using three items each (six items total) similar to those in the sibling relations subscale ("does things with mother/father", “is friendly/affectionate toward mother/father", "talks with mother/father"). The parent relations subscale also contains several items assessing problems with parents (e.g., "has strong negative reaction or refuses to do chores or honor restrictions", "damages home or family property").

\footnotetext{
2 One item ("wants to be with girls/boys [opposite sex]") was excluded from the peer functioning subscale due to a negative correlation ( $r \mathrm{~s}=-.01$ and -.08$)$ with the subscale score.

3 One parent relations item ("damages home or family property") was excluded because $97 \%$ of adolescents and $99 \%$ of parents scored this item as 'not a problem.'
} 
Adolescents' functioning was assessed using both self- and parent-report for all but six participants. These six participants (five of whom were singletons) were missing self-reported peer dysfunction data, and thus were excluded from analyses of self-reported (but not parentreported) adolescent peer dysfunction. The parent-report SAICA was completed by the mother for $90.1 \%$ of participants. Internal consistency was adequate for the peer dysfunction (self-report $\alpha=.73$; parent-report $\alpha=.83$ ) and dysfunction in sibling (self-report $\alpha=.80$; parent-report $\alpha=.78$ ) and parent (self-report $\alpha=.75$; parent-report $\alpha=.80$ ) relations subscales.

\section{Depressive Symptoms}

Depressive symptoms in adolescence were included as a covariate in certain analyses and was measured using the Center for Epidemiological Studies Depression Scale for Children (CESDC; Weissman et al., 1980), a version of the Center for Epidemiologic Studies Depression Scale (CES-D; Radloff, 1977) modified for children and adolescents. The internal consistency of the CES-DC was acceptable $(\alpha=.73)$. Depressive symptoms in adulthood were assessed at up to five follow-ups spaced approximately five years apart, and were measured using the CES-D at the first follow-up ( $\alpha=.92$ ), the Hamilton Rating Scale for Depression (HRSD; Hamilton, 1960) at the second $(\alpha=.83)$ and third follow-ups $(\alpha=.92)$, and the Patient Health Questionnaire (PHQ-9; Kroenke et al., 2001) at the fourth $(\alpha=.92)$ and fifth $(\alpha=.81)$ follow-ups. Participants completed only one measure of depressive symptoms at each follow-up. Although the variation in depressive symptom measures is suboptimal and the inclusion of only one scale at each followup precluded the calculation of contemporaneous cross-measure correlations, previous studies indicate these measures are moderately to highly intercorrelated and have a great deal of content overlap (e.g., Chin et al., 2015; Sun et al., 2020). The five adult depressive symptom assessments were z-scored within wave prior to analyses. 


\section{Data Analysis}

As adult depressive symptoms were assessed up to five times per person, the data had a three-level structure with adult depressive symptom assessments (level 1) clustered within individuals (level 2), who in turn were clustered within families (level 3). Multilevel modeling was used to estimate associations between peer dysfunction and adult depressive symptoms while accounting for the non-independence of observations. Although several statistical approaches can estimate within-family effects in sibling data (e.g., fixed effect analysis), multilevel modeling was used because it can more easily handle three-level data. This allowed us to directly model level 1 adult depressive symptom scores, which is preferable to modeling aggregates across level 1 (e.g., person-level averages of adult depressive symptom scores) for several reasons. First, aggregating across level 1 would assume zero within-person variability in adult depressive symptoms, whereas modeling level 1 observations in a multilevel model explicitly models within-person variability (Clarke, 2008). Second, multilevel modeling accounts for between-person variability in the amount of missing adult depressive symptom data by weighting parameter estimates such that participants with less missing data have stronger influences on parameter estimates (Snijders \& Bosker, 2012), thereby allowing all nonmissing depressive symptom assessments in adulthood to be included in the models. All models used maximum likelihood estimation assuming missingness at random and included nested random intercepts at the individual and family levels. The effects of peer dysfunction and covariates (when included) were modeled as person-level (level 2) fixed effect predictors of the random person-level intercept. Random slopes were not included there were too few siblings per family to reliably identify estimate both random intercepts and slopes (Singmann \& Kellen, 2019). The 
effects of self- and parent-reported adolescent peer dysfunction were examined separately in the primary analyses because the inter-rater correlation was only moderate, $r=.51, p<.001$.

The association between peer dysfunction in adolescence and depressive symptoms in adulthood was examined in four models with increasingly strict statistical and/or methodological controls. First, we estimated the unadjusted association between adolescent peer dysfunction and adult depressive symptoms. Second, potential individual-level confounds were added as statistical covariates. Sex, age, and dysfunctional relations with parents or siblings in adolescence were included as individual-level covariates due to their associations with depression in previous studies (Avenevoli et al., 2015; Landstedt et al., 2015; Waldinger et al., 2007). Of note, covarying for adolescent social functioning in non-peer domains (i.e., parents, siblings) tested whether the association between peer dysfunction and adult depressive symptoms was independent of dysfunction in non-peer relationships. We also covaried for depression symptoms in adolescence to rule out the confounding effect of depressive symptoms in adolescence. Potential family-level confounds such as family history of MDD were not included as statistical covariates because they were methodologically controlled for in subsequent models using sibling comparison. Indeed, the main strength of the sibling comparison design is its ability to rule out the confounding effects of all characteristics shared between siblings. Controlling for either select individual-level characteristics or unmeasured familial characteristics in isolation and then controlling for both sets of confounders simultaneously allows the opportunity to quantify the extent to which the association is confounded by select individual-level characteristics versus family-level characteristics. 
Third, we used sibling comparison to test whether the association was due to familial confounding. The mean of peer dysfunction scores was first calculated for each family, ${ }^{4}$ and serves as a proxy for family-level genetic and environmental factors that are correlated with adolescent peer dysfunction. We then created a family-centered peer dysfunction variable representing each sibling's deviation (i.e., discordance) from their family's mean. This familycentered variable reflects the amount of peer dysfunction relative to the mean peer dysfunction of all adolescents in the family. For example, if a family's mean was 5 and a sibling within that family had a score of 9 , that sibling's family-centered score would be 9 minus 5 , or 4 . These calculations are demonstrated in Table S1 in Online Resource 1. Finally, the peer dysfunction predictor in the first unadjusted model was replaced in this model by the family-centered peer dysfunction score. The effect of family-centered peer dysfunction is the within-family effect, which is a more stringent test of causality because it controls for all genetic and environmental confounders shared among siblings (D'Onofrio et al., 2007). If the association between adolescent peer dysfunction and adult depressive symptoms is entirely due to confounding factors shared between siblings (e.g., MDD family history), one would expect all siblings that share these factors to have similar adult depressive symptom scores. In this scenario, the association would be reduced to approximately zero when comparing siblings. In contrast, if family-level factors have no confounding effect, one would expect the association to be relatively unchanged when comparing siblings. Singletons $(n=15)$ were excluded from all models involving sibling comparison and when calculating family-centered peer dysfunction scores.

\footnotetext{
${ }^{4}$ Family-level means of adolescent peer dysfunction were calculated using all individuals with adolescent peer dysfunction data (even if they had no adult depression data) to maximize the reliability of these estimates, and were based on $2.64(S D=0.59)$ and $2.60(S D=0.61)$ adolescents per family for parent- and self-reported adolescent peer dysfunction, respectively.
} 
Fourth, we combined the use of sibling comparison and measured covariates by simultaneously entering the family-centered peer dysfunction score as a predictor (as was done in the third model) and including the same set of measured covariates from the second model. This approach controls for both measured individual-level confounders and unmeasured familial confounds.

Lastly, supplemental analyses tested whether prospective associations were specific to either self-reported or parent-reported peer dysfunction by re-estimating the four models described above with both self- and parent-reported peer dysfunction included as simultaneous predictors. As in the primary analyses described above, family-centered peer dysfunction scores were used in the two models using sibling comparison and raw (i.e., uncentered) peer dysfunction scores were used in the other two models. Analyses were performed in R using the lme4 (Bates et al., 2015), lmerTest (Kuznetsova et al., 2017), and simr (Green \& Macleod, 2016) packages.

\section{Statistical Power.}

Statistical power in sibling comparison studies is related to the amount of within-family variability (e.g., discordance) in the exposure (Li et al., 2014). The average within-family ranges for self-reported $(M=0.25)$ and parent-reported $(M=0.31)$ peer dysfunction scores were equivalent to 0.97 SDs and 1.21 SDs, respectively, indicating sufficient within-family variation in peer dysfunction (Kim, 2021). The overall distributions of the family-centered peer dysfunction variables also indicated sufficient within-family variability for both self-reported $(M=-0.01, S D=0.16$, range $=-0.44-0.49)$ and parent-reported peer dysfunction $(M=0.00, S D=0.21$, range $=-0.67-0.67)$. 
A post-hoc sensitivity analysis was also conducted using Monte Carlo simulations. We substituted the effect of adolescent peer dysfunction with effect sizes ranging from .20 to 1.20 in increments of .10. For each effect size, we simulated each model 500 times and then extracted the proportion of iterations for which the effect of adolescent peer dysfunction on adult depressive symptoms was statistically significant (i.e., power) at alpha $=.05$. As in all other analyses, singletons were excluded from sensitivity analyses involving sibling comparison. The resulting power curves are plotted in Figure S1 in Online Resource 1.

\section{Results}

\section{Preliminary Analyses}

Correlations among individual characteristics in adolescence are presented in Figure 1. Preliminary analyses examined whether these characteristics differed between (a) individuals who completed at least one assessment of depressive symptoms in adulthood and individuals who did not (and were thus excluded from all analyses), or (b) singletons and non-singletons. Individuals who completed at least one depressive symptom assessment in adulthood did not differ from individuals who did not ( $p s>$.148). Additionally, singletons and non-singletons did not differ on any baseline characteristics or depression symptom severity in adulthood ( $p s>.588)$, supporting the generalizability of results from sibling comparison models to singletons (Lahey \& D’Onofrio, 2010). Lastly, assumptions of multilevel modeling were examined using statistical tests and visualizations. Breusch-Pagan tests (Breusch \& Pagan, 1979) indicated that residual variances were homogeneous $(p>.05$ for all models; also see Figure S3 in the supplementary materials). Diagnostic plots also indicated that the assumptions of normality of residuals and linearity were generally met (see Figures S4 and S5 in the supplementary materials).

\section{Association Between Adolescent Peer Dysfunction and Adult Depression}


Unstandardized coefficients from models testing the association between adolescent peer dysfunction and adult depressive symptoms are presented in Figure 2 and Tables 2 and 3 . The positive unadjusted association for self-reported adolescent peer dysfunction $(b=1.28, p<.001)$ was consistent with findings from unrelated individuals (e.g., Bagwell et al., 2001; Bean et al., 2019; Landstedt et al., 2015; Modin et al., 2011), and was not attenuated when controlling for sex and other individual characteristics (i.e., depressive symptoms, age, and dysfunction in relations with siblings and parents) in adolescence $(b=1.34, p=.003)$. The association remained significant in the sibling comparison model that controlled for familial confounding $(b=1.15$, $p=.005)$. The sibling comparison model that additionally controlled for measured covariates also indicated a significant (albeit slightly reduced) association $(b=1.06, p=.035)$.

Analyses of parent-reported adolescent peer dysfunction similarly found a significant unadjusted association with adult depressive symptoms $(b=0.56, p=.032)$, although this effect was substantially weaker than the unadjusted association for self-reported peer dysfunction. This association was further weakened and no longer significant $(b s \leq 0.41, p s \geq .182)$ after introducing statistical (i.e., measured covariates) and/or methodological (i.e., sibling comparison) controls.

Results of supplemental analyses examining the unique effects of self- versus parentreported adolescent peer dysfunction are plotted in Figure S2 in Online Resource 2. Self-reported peer dysfunction significantly predicted adult depressive symptoms in all four models $(b \mathrm{~s} \geq 1.28$, $p s \leq .013)$ and its unique association was not attenuated by the inclusion of statistical covariates and/or use of sibling comparison. In contrast, parent-reported peer dysfunction did not uniquely predict adult depressive symptoms in any of the models ( $b \mathrm{~s} \leq 0.00, p \mathrm{~s} \geq .199)$.

\section{Discussion}


Preventing depression is a pressing public health issue, and peer dysfunction in adolescence predicts depression in adulthood. Determining whether this effect is due to confounders is a critical requisite to effectively reduce depression risk through targeted prevention. This is particularly urgent considering the prevalence of adolescent peer dysfunction (e.g., one-third of adolescents report being bullied by peers; Modecki et al., 2014) and dramatic reductions in youth's face-to-face peer contact and support during the COVID-19 pandemic (Orben et al., 2020; Rogers et al., 2021), potentially increasing risk for depression. The present study examined the prospective association between peer dysfunction in adolescence and depressive symptoms in adulthood, and compared siblings within families to test whether this association remained after adjusting for family-level confounders. We found that both self- and parent-reported adolescent peer dysfunction predicted adult depressive symptoms up to 38 years later, replicating prior longitudinal studies of unrelated individuals (Bagwell et al., 2001; Bean et al., 2019; Copeland et al., 2013; Landstedt et al., 2015; Modin et al., 2011). Results further indicated that the prospective association between self-reported adolescent peer dysfunction and adult depressive symptoms was only slightly attributable to unmeasured genetic and environmental confounders or measured covariates (e.g., baseline depressive symptoms and dysfunction in sibling and parent relations in adolescence). Sibling comparison is a strong quasiexperimental design, and these results suggest that self-reported peer dysfunction may be an unconfounded risk factor for depressive symptoms in adulthood. The effect of parent-reported adolescent peer dysfunction on adult depressive symptoms was attenuated and no longer significant when controlling for baseline individual-level covariates or unmeasured familial confounding. 
Peer dysfunction and depression in adolescence are interwoven with numerous potentially confounding characteristics within the individual and family (Deater-Deckard, 2001). This study ruled out the confounding effects of familial characteristics and several important individual-level characteristics (e.g., adolescent depressive symptoms and dysfunction in sibling and parent relations), which extends previous studies of unrelated individuals (Bagwell et al., 2001; Bean et al., 2019; Copeland et al., 2013; Landstedt et al., 2015; Modin et al., 2011) by controlling for a much more comprehensive set of potential confounders and more strongly testing the potentially causal relationship between adolescent peer dysfunction and adult depressive symptoms. Supplemental analyses including self- and parent-reported peer dysfunction as simultaneous predictors found that the effect of self-reported peer dysfunction was largely independent of parent-reported peer dysfunction, suggesting specificity. This finding is consistent with evidence that it is the adolescent's perception of peer dysfunction - not peer dysfunction as perceived by parents, teachers, or peers - that increases risk for depressive symptoms (Epkins \& Seegan, 2015; Kistner et al., 1999). However, the sibling comparison design cannot demonstrate causality because it does not rule out potential confounders that differ between siblings (Frisell et al., 2012). For example, interpersonal theories of depression suggest that interpersonal skills deficits (e.g., excessive reassurance seeking) or impulse control difficulties contribute to peer rejection and the onset, maintenance, or exacerbation of depressive symptoms (Coyne, 1976; Gorka et al., 2013; Humphreys et al., 2013). Residual genetic confounding is also possible, as genetic factors that differed between siblings might influence both peer dysfunction and risk for depression. Comparing monozygotic twins can rule out all genetic confounders and represents a future direction for extending this work. Additionally, inferences from sibling comparison designs can be biased if an individual's adolescent peer 
dysfunction or adult depression impacts those of their sibling(s) (Sjölander et al., 2016). Finally, sibling comparison results can only be generalized to singletons if siblings do not meaningfully differ from singletons in the population (Lahey \& D’Onofrio, 2010). Siblings and singletons did not significantly differ on a variety of characteristics in the present study, suggesting this assumption may be satisfied. However, the relatively few singletons $(n=15)$ may not be representative of singletons in the population. In light of these limitations of the sibling comparison design, triangulation with other designs with different limitations may help to support stronger causal inferences regarding the effect of dysfunction with peers on adult depression (Lawlor et al., 2016).

Keeping these caveats in mind, these findings support self-reported adolescent peer dysfunction as a potentially "causal" risk factor for depressive symptoms in adulthood. This inference is consistent with the results of a retrospective twin study of self-reported adolescent peer victimization and depressive episodes in adulthood (Kretschmer et al., 2018), which found that this association could only partially be explained by genetic and environmental confounding. Although several non-causal explanations for this association cannot be ruled out (as discussed above), there are several possible mediating pathways underlying this relationship. Cognitive theories of depression posit that the perception of prolonged peer dysfunction may decrease selfesteem (e.g., Fenzel, 2000), leading to the development of negative inferential styles and the belief that desired interpersonal outcomes are unattainable (Panzarella et al., 2006; Rose \& Abramson, 1992). In turn, negative inferential styles may interact with negative life events later in adolescence or adulthood to engender hopelessness, which may cause depression either directly (Abramson et al., 1989) or through decreased goal-directed behavior (Davidson, 1998; McFarland et al., 2006). 
If causal, these findings have several implications for clinical practice and prevention. They suggest that preventative interventions could directly reduce depression risk in adulthood by reducing peer dysfunction in adolescence. Strong tests of causation such as these are important precursors to the development of interventions based on causal mechanisms underlying risk (Cuijpers et al., 2012). Successful reduction of adolescent peer dysfunction may also prevent the development of maladaptive processes mediating the relationship between peer dysfunction and depression (e.g., negative inferential style), obviating the need to target these mediators for prevention. Results also have implications for screening and resource allocation in the context of prevention. Selective or indicated prevention programs offered to adolescents at elevated depression risk are more efficacious than universal programs, and risk status has typically been determined using elevated depression symptom severity, negative inferential style, parental mood disorders, or familial conflict (Stice et al., 2009). Pending replication and extension, these findings suggest that using peer dysfunction as an important indicator of risk could help to identify high-risk adolescents who would benefit most from preventative intervention.

It is important to note that peer dysfunction encapsulates a wide range of dimensions and behaviors (e.g., trouble making friends, victimization, unpopularity, lack of close friendships) and the observed associations between adolescent peer dysfunction and adult depressive symptoms could be specific to certain aspects of peer dysfunction. Different dimensions of peer dysfunction are differentially associated with interpersonal skills, competencies, and outcomes (Asher \& Weeks, 2018). For example, one study found that the strength of dyadic friendships (but not broader popularity) in mid-adolescence predicted depressive symptoms in early adulthood (Narr et al., 2019). The heterogeneity of the peer dysfunction measure thus prevents 
more specific insights and hypotheses regarding both (a) potential mechanistic pathway(s) from adolescent peer dysfunction to adult depressive symptoms, and (b) preventative interventions that target specific domains of peer dysfunction. That is, preventative interventions are unlikely to reduce all dimensions of peer dysfunction equally and identifying more specific intervention targets would inform intervention selection. For these reasons, testing whether these findings are specific to particular dimensions of peer dysfunction is a critical direction for future research.

Compared to self-reported adolescent peer dysfunction, parent-reported adolescent peer dysfunction was more weakly associated with adult depressive symptoms across all models. The association between parent-reported peer dysfunction and adult depressive symptoms also became nonsignificant when controlling for individual-level covariates, family-level confounders (e.g., MDD family history), and/or self-reported peer dysfunction. This suggests that the association for parent-reported peer dysfunction may be due to confounders. However, confidence intervals were wide and sensitivity analyses indicated that the nonsignificant effect of parent-reported peer dysfunction when using methodological and/or statistical controls may have been a type II error. Thus, the extent to which the effect of parent-reported peer dysfunction is due to confounders is unclear.

This study had several noteworthy strengths, including (a) the use of a quasiexperimental and longitudinal design that tested prospective associations across a long follow-up while controlling for a variety of confounders, (b) the examination of both self- and parentreported adolescent peer dysfunction as assessed by semi-structured interview, and (c) consideration of dysfunction in relations with siblings and parents as potential confounds. There were also several notable limitations. First, confidence intervals were wide due to the relatively small sample size, particularly when evaluating the magnitude of attenuation caused by adding 
certain controls. Results should therefore be considered preliminary until they are replicated in larger samples. Second, the sample was entirely white. Recruiting racially homogeneous samples was unfortunately standard practice when data collection began in the 1980s, and it is critical that future studies examine generalizability in other racial/ethnic groups. Studying generalizability in marginalized groups is particularly important so that improvements in knowledge regarding etiology and clinical practice do not disproportionately apply to or benefit privileged groups. Third, depressive symptoms in adulthood were assessed using three different measures that, although moderately to highly correlated (e.g., Chin et al., 2015; Sun et al., 2020), are not identical in content (Fried, 2017). Fourth, the inclusion of a disproportionately high number of individuals with a family history of MDD likely increased statistical power by increasing variability in adult depression scores, but may impact generalizability to more population-based samples.

\section{Conclusion}

These results suggest that the prospective association between perceived peer dysfunction in adolescence and depressive symptoms in adulthood cannot be explained by a variety of potential confounds, suggesting that the association may be direct. If replicated and extended in larger samples, these findings provide support for targeting adolescent's perceptions of peer dysfunction to reduce depressive symptoms in adulthood. 


\section{References}

Abramson, L. Y., Metalsky, G. I., \& Alloy, L. B. (1989). Hopelessness depression: A theorybased subtype of depression. Psychological Review, 96(2), 358-372. https://doi.org/10.1037/0033-295X.96.2.358

Asher, S. R., \& Weeks, M. S. (2018). Friendships in Childhood. In The Cambridge Handbook of Personal Relationships (pp. 119-134). Cambridge University Press. https://doi.org/10.1017/9781316417867.011

Avenevoli, S., Swendsen, J., He, J. P., Burstein, M., \& Merikangas, K. R. (2015). Major Depression in the National Comorbidity Survey-Adolescent Supplement: Prevalence, Correlates, and Treatment. Journal of the American Academy of Child and Adolescent Psychiatry, 54(1), 37-44.e2. https://doi.org/10.1016/j.jaac.2014.10.010

Bagwell, C. L., Schmidt, M. E., Newcomb, A. F., \& Bukowski, W. M. (2001). Friendship and peer rejection as predictors of adult adjustment. New Directions for Child and Adolescent Development, 91, 25-49. https://doi.org/10.1002/cd.4

Ball, H. A., Arseneault, L., Taylor, A., Maughan, B., Caspi, A., \& Moffitt, T. E. (2008). Genetic and environmental influences on victims, bullies and bully-victims in childhood. Journal of Child Psychology and Psychiatry, 49(1), 104-112. https://doi.org/10.1111/j.14697610.2007.01821.x

Bates, D., Mächler, M., Bolker, B. M., \& Walker, S. C. (2015). Fitting linear mixed-effects models using lme4. Journal of Statistical Software, 67(1). https://doi.org/10.18637/jss.v067.i01

Bean, C. G., Pingel, R., Hallqvist, J., Berg, N., \& Hammarström, A. (2019). Poor peer relations in adolescence, social support in early adulthood, and depressive symptoms in later 
ADOLESCENT PEER DYSFUNCTION ADULT DEPRESSION 23

adulthood-Evaluating mediation and interaction using four-way decomposition analysis. Annals of Epidemiology, 29, 52-59. https://doi.org/10.1016/j.annepidem.2018.10.007

Boivin, M., Brendgen, M., Vitaro, F., Dionne, G., Girard, A., Pérusse, D., \& Tremblay, R. E. (2013). Strong Genetic Contribution to Peer Relationship Difficulties at School Entry: Findings From a Longitudinal Twin Study. Child Development, 84(3), 1098-1114. https://doi.org/10.1111/cdev.12019

Bowes, L., Joinson, C., Wolke, D., \& Lewis, G. (2015). Peer victimisation during adolescence and its impact on depression in early adulthood: Prospective cohort study in the United Kingdom. BMJ (Online), 350, 2469. https://doi.org/10.1136/bmj.h2469

Brendgen, M., Ouellet-Morin, I., Lupien, S., Vitaro, F., Dionne, G., \& Boivin, M. (2017). Does cortisol moderate the environmental association between peer victimization and depression symptoms? A genetically informed twin study. Psychoneuroendocrinology, 84, 42-50. https://doi.org/10.1016/J.PSYNEUEN.2017.06.014

Breusch, T. S., \& Pagan, A. R. (1979). A Simple Test for Heteroscedasticity and Random Coefficient Variation. Econometrica, 47(5), 1287-1294. https://doi.org/10.2307/1911963

Chin, W. Y., Choi, E. P. H., Chan, K. T. Y., \& Wong, C. K. H. (2015). The Psychometric Properties of the Center for Epidemiologic Studies Depression Scale in Chinese Primary Care Patients: Factor Structure, Construct Validity, Reliability, Sensitivity and Responsiveness. PLOS ONE, 10(8), e0135131. https://doi.org/10.1371/journal.pone.0135131

Clarke, P. (2008). When can group level clustering be ignored? Multilevel models versus singlelevel models with sparse data. Journal of Epidemiology and Community Health, 62(8), 752-758. https://doi.org/10.1136/jech.2007.060798 
Cohen, S., \& Wills, T. A. (1985). Stress, Social Support, and the Buffering Hypothesis. Psychological Bulletin, 98(2), 310-357. https://doi.org/10.1037/0033-2909.98.2.310

Copeland, W. E., Wolke, D., Angold, A., \& Costello, E. J. (2013). Adult psychiatric outcomes of bullying and being bullied by peers in childhood and adolescence. JAMA Psychiatry, 70(4), 419-426. https://doi.org/10.1001/jamapsychiatry.2013.504

Coyne, J. C. (1976). Toward an Interactional Description of Depression. Psychiatry, 39(1), 2840. https://doi.org/10.1080/00332747.1976.11023874

Cuijpers, P., Beekman, A. T. F., \& Reynolds, C. F. (2012). Preventing depression: A global priority. JAMA, 307(10), 1033-1034. https://doi.org/10.1001/jama.2012.271

Davidson, R. J. (1998). Affective Style and Affective Disorders: Perspectives from Affective Neuroscience. Cognition and Emotion, 12(3), 307-330. https://doi.org/10.1080/026999398379628

Deater-Deckard, K. (2001). Annotation: Recent Research Examining the Role of Peer Relationships in the Development of Psychopathology. Journal of Child Psychology and Psychiatry, 42(5), 565-579. https://doi.org/10.1111/1469-7610.00753

D’Onofrio, B. M., Van Hulle, C. A., Waldman, I. D., Rodgers, J. L., Rathouz, P. J., \& Lahey, B. B. (2007). Causal inferences regarding prenatal alcohol exposure and childhood externalizing problems. Archives of General Psychiatry, 64(11), 1296-1304. https://doi.org/10.1001/archpsyc.64.11.1296

Epkins, C. C., \& Seegan, P. L. (2015). Mother-Reported and Children's Perceived Social and Academic Competence in Clinic-Referred Youth: Unique Relations to Depression and/or Social Anxiety and the Role of Self-Perceptions. Child Psychiatry and Human Development, 46(5), 656-670. https://doi.org/10.1007/s10578-014-0508-9 
Fenzel, L. M. (2000). Prospective study of changes in global self-worth and strain during the transition to middle school. Journal of Early Adolescence, 20(1), 93-116. https://doi.org/10.1177/0272431600020001005

Fried, E. I. (2017). The 52 symptoms of major depression: Lack of content overlap among seven common depression scales. Journal of Affective Disorders, 208, 191-197. https://doi.org/10.1016/j.jad.2016.10.019

Friedrich, M. J. (2017). Depression is the leading cause of disability around the world. JAMA, 317(15), 1517. https://doi.org/10.1001/jama.2017.3826

Frisell, T. (2020). Sibling comparison designs, are they worth the effort? American Journal of Epidemiology. https://doi.org/10.1093/aje/kwaa183

Frisell, T., Ã-Berg, S., Kuja-Halkola, R., \& Sjölander, A. (2012). Sibling comparison designs: Bias from non-shared confounders and measurement error. Epidemiology, 23(5), 713720. https://doi.org/10.1097/EDE.0b013e31825fa230

Garber, J., \& Cole, D. A. (2010). Intergenerational transmission of depression: A launch and grow model of change across adolescence. Development and Psychopathology, 22(4), 819-830. https://doi.org/10.1017/S0954579410000489

Gjerde, L. C., Eilertsen, E. M., Reichborn-Kjennerud, T., McAdams, T. A., Zachrisson, H. D., Zambrana, I. M., Røysamb, E., Kendler, K. S., \& Ystrom, E. (2017). Maternal perinatal and concurrent depressive symptoms and child behavior problems: A sibling comparison study. Journal of Child Psychology and Psychiatry, 58(7), 779-786. https://doi.org/10.1111/jcpp.12704 
Goodman, S. H., Brogan, D., Lynch, M. E., \& Fielding, B. (1993). Social and Emotional Competence in Children of Depressed Mothers. Child Development, 64(2), 516-531. https://doi.org/10.1111/j.1467-8624.1993.tb02925.x

Goodman, S. H., \& Gotlib, I. H. (1999). Risk for psychopathology in the children of depressed mothers: A developmental model for understanding mechanisms of transmission. Psychological Review, 106(3), 458-490. https://doi.org/10.1037/0033-295X.106.3.458

Gorka, S. M., Shankman, S. A., Seeley, J. R., \& Lewinsohn, P. M. (2013). The moderating effect of parental illicit substance use disorders on the relation between adolescent depression and subsequent illicit substance use disorders. Drug and Alcohol Dependence, 128(1-2), 1-7. https://doi.org/10.1016/j.drugalcdep.2012.07.011

Green, P., \& Macleod, C. J. (2016). SIMR: An R package for power analysis of generalized linear mixed models by simulation. Methods in Ecology and Evolution, 7(4), 493-498. https://doi.org/10.1111/2041-210X.12504

Hamilton, M. (1960). A Rating Scale for Depression. Journal of Neurology, Neurosurgery, and Psychiatry, 23(1), 56-62.

Hammen, C. (2005). Stress and depression. Annual Review of Clinical Psychology, 1, 293-319. https://doi.org/10.1146/annurev.clinpsy.1.102803.143938

Hammen, C., Shih, J. H., \& Brennan, P. A. (2004). Intergenerational transmission of depression: Test of an interpersonal stress model in a community sample. Journal of Consulting and Clinical Psychology, 72(3), 511-522. https://doi.org/10.1037/0022-006X.72.3.511

Humphreys, K. L., Katz, S. J., Lee, S. S., Hammen, C., Brennan, P. A., \& Najman, J. M. (2013). The association of ADHD and depression: Mediation by peer problems and parent-child 
difficulties in two complementary samples. Journal of Abnormal Psychology, 122(3), 854-867. https://doi.org/10.1037/a0033895

John, K., Gammon, D. G., Prusoff, B. A., \& Warner, V. (1987). The Social Adjustment Inventory for Children and Adolescents (SAICA): Testing of a New Semistructured Interview. Journal of the American Academy of Child and Adolescent Psychiatry, 26(6), 898-911. https://doi.org/10.1097/00004583-198726060-00015

Kim, J. (2021). The quality of social relationships in schools and adult health: Differential effects of student-student versus student-teacher relationships. School Psychology, 36(1), 6-16. https://doi.org/10.1037/spq0000373

Kistner, J., Balthazor, M., Risi, S., \& Burton, C. (1999). Predicting Dysphoria in Adolescence from Actual and Perceived Peer Acceptance in Childhood. Journal of Clinical Child and Adolescent Psychology, 28(1), 94-104. https://doi.org/10.1207/s15374424jccp2801_8

Kochel, K. P., Ladd, G. W., \& Rudolph, K. D. (2012). Longitudinal Associations Among Youth Depressive Symptoms, Peer Victimization, and Low Peer Acceptance: An Interpersonal Process Perspective. Child Development, 83(2), 637-650. https://doi.org/10.1111/j.14678624.2011.01722.x

Kretschmer, T., Tropf, F. C., \& Niezink, N. M. D. (2018). Causality and Pleiotropy in the Association Between Bullying Victimization in Adolescence and Depressive Episodes in Adulthood. Twin Research and Human Genetics, 21(1), 33-41. https://doi.org/10.1017/thg.2017.71

Kroenke, K., Spitzer, R. L., \& Williams, J. B. (2001). The PHQ-9: Validity of a brief depression severity measure. Journal of General Internal Medicine, 16(9), 606-613. https://doi.org/10.1046/J.1525-1497.2001.016009606.X 
Kuznetsova, A., Brockhoff, P. B., \& Christensen, R. H. B. (2017). lmerTest Package: Tests in Linear Mixed Effects Models. Journal of Statistical Software, 82(13). https://doi.org/10.18637/jss.v082.i13

Lahey, B. B., \& D’Onofrio, B. M. (2010). All in the family: Comparing siblings to test causal hypotheses regarding environmental influences on behavior. Current Directions in Psychological Science, 19(5), 319-323. https://doi.org/10.1177/0963721410383977

Landstedt, E., Hammarstrom, A., \& Winefield, H. (2015). How well do parental and peer relationships in adolescence predict health in adulthood? Scandinavian Journal of Public Health, 43(5), 460-468. https://doi.org/10.1177/1403494815576360

Larson, R. W., Moneta, G., Richards, M. H., Holmbeck, G., \& Duckett, E. (1996). Changes in adolescents' daily interactions with their families from ages 10 to 18: Disengagement and transformation. Developmental Psychology, 32(4), 744-754.

https://doi.org/10.1037/0012-1649.32.4.744

Lawlor, D. A., Tilling, K., \& Smith, G. D. (2016). Triangulation in aetiological epidemiology. International Journal of Epidemiology, 45(6), 1866-1886. https://doi.org/10.1093/ije/dyw314

Li, Z., Mckeague, I. W., \& Lumey, L. H. (2014). Optimal design strategies for sibling studies with binary exposures. International Journal of Biostatistics, 10(2), 185-196. https://doi.org/10.1515/ijb-2014-0015

Mannuzza, S., Fyer, A. J., Klein, D. F., \& Endicott, J. (1986). Schedule for Affective Disorders and Schizophrenia-Lifetime version modified for the study of anxiety disorders (SADSLA): Rationale and conceptual development. Journal of Psychiatric Research, 20(4), 317-325. https://doi.org/10.1016/0022-3956(86)90034-8 
McFarland, B. R., Shankman, S. A., Tenke, C. E., Bruder, G. E., \& Klein, D. N. (2006). Behavioral activation system deficits predict the six-month course of depression. Journal of Affective Disorders, 91(2-3), 229-234. https://doi.org/10.1016/j.jad.2006.01.012

Michelini, G., Perlman, G., Tian, Y., Mackin, D. M., Nelson, B. D., Klein, D. N., \& Kotov, R. (2021). Multiple domains of risk factors for first onset of depression in adolescent girls. Journal of Affective Disorders, 283, 20-29. https://doi.org/10.1016/j.jad.2021.01.036

Modecki, K. L., Minchin, J., Harbaugh, A. G., Guerra, N. G., \& Runions, K. C. (2014). Bullying prevalence across contexts: A meta-analysis measuring cyber and traditional bullying. In Journal of Adolescent Health (Vol. 55, Issue 5, pp. 602-611). Elsevier USA. https://doi.org/10.1016/j.jadohealth.2014.06.007

Modin, B., Östberg, V., \& Almquist, Y. (2011). Childhood peer status and adult susceptibility to anxiety and depression: A 30-year hospital follow-up. Journal of Abnormal Child Psychology, 39(2), 187-199. https://doi.org/10.1007/s10802-010-9462-6

Morneau-Vaillancourt, G., Dionne, G., Brendgen, M., Vitaro, F., Feng, B., Henry, J., ForgetDubois, N., Tremblay, R., \& Boivin, M. (2019). The Genetic and Environmental Etiology of Shyness Through Childhood. Behavior Genetics, 49(4), 376-385. https://doi.org/10.1007/s10519-019-09955-w

Narr, R. K., Allen, J. P., Tan, J. S., \& Loeb, E. L. (2019). Close Friendship Strength and Broader Peer Group Desirability as Differential Predictors of Adult Mental Health. Child Development, 90(1), 298-313. https://doi.org/10.1111/cdev.12905

Orben, A., Tomova, L., \& Blakemore, S. J. (2020). The effects of social deprivation on adolescent development and mental health. In The Lancet Child and Adolescent Health 
(Vol. 4, Issue 8, pp. 634-640). Elsevier B.V. https://doi.org/10.1016/S23524642(20)30186-3

Panzarella, C., Alloy, L. B., \& Whitehouse, W. G. (2006). Expanded hopelessness theory of depression: On the mechanisms by which social support protects against depression. Cognitive Therapy and Research, 30(3), 307-333. https://doi.org/10.1007/s10608-0069048-3

Radloff, L. S. (1977). The CES-D scale: A self-report depression scale for research in the general population. Applied Psychological Measurement, 1(3), 385-401.

Reijntjes, A., Kamphuis, J. H., Prinzie, P., \& Telch, M. J. (2010). Peer victimization and internalizing problems in children: A meta-analysis of longitudinal studies. Child Abuse and Neglect, 34(4), 244-252. https://doi.org/10.1016/j.chiabu.2009.07.009

Reinherz, H. Z., Paradis, A. D., Giaconia, R. M., Stashwick, C. K., \& Fitzmaurice, G. (2003). Childhood and adolescent predictors of major depression in the transition to adulthood. American Journal of Psychiatry, 160(12), 2141-2147. https://doi.org/10.1176/appi.ajp.160.12.2141

Roach, A. (2018). Supportive Peer Relationships and Mental Health in Adolescence: An Integrative Review. Issues in Mental Health Nursing, 39(9), 723-737. https://doi.org/10.1080/01612840.2018.1496498

Rogers, A. A., Ha, T., \& Ockey, S. (2021). Adolescents' Perceived Socio-Emotional Impact of COVID-19 and Implications for Mental Health: Results From a U.S.-Based MixedMethods Study. Journal of Adolescent Health, 68(1), 43-52. https://doi.org/10.1016/j.jadohealth.2020.09.039 
Rose, D. T., \& Abramson, L. Y. (1992). Developmental predictors of depressive cognitive style: Research and theory. In D. Cicchetti \& S. L. Toth (Eds.), Rochester symposium of developmental psychopathology (pp. 323-349). Earlbaum.

Rudolph, K. D. (2009). The interpersonal context of adolescent depression. In S. NolenHoeksema \& L. M. Hilt (Eds.), Handbook of depression in adolescents (pp. 377-418). Routledge/Taylor \& Francis Group.

Shapero, B. G., Hankin, B. L., \& Barrocas, A. L. (2013). Stress generation and exposure in a multi-wave study of adolescents: Transactional processes and sex differences. Journal of Social and Clinical Psychology, 32(9), 989-1012. https://doi.org/10.1521/jscp.2013.32.9.989

Silberg, J. L., Maes, H., \& Eaves, L. J. (2010). Genetic and environmental influences on the transmission of parental depression to children's depression and conduct disturbance: An extended Children of Twins study. Journal of Child Psychology and Psychiatry and Allied Disciplines, 51(6), 734-744. https://doi.org/10.1111/j.1469-7610.2010.02205.x

Singmann, H., \& Kellen, D. (2019). An introduction to mixed models for experimental psychology. In New Methods in Cognitive Psychology (pp. 4-31). Routledge. https://doi.org/10.4324/9780429318405-2

Sjölander, A., Frisell, T., Kuja-Halkola, R., Öberg, S., \& Zetterqvist, J. (2016). Carryover effects in sibling comparison designs. Epidemiology, 27(6), 852-858. https://doi.org/10.1097/EDE.0000000000000541

Snijders, T. A. B., \& Bosker, R. J. (2012). Multilevel analysis: An introduction to basic and advanced multilevel modeling (2nd ed.). SAGE Publications. 
Stice, E., Shaw, H., Bohon, C., Marti, C. N., \& Rohde, P. (2009). A Meta-Analytic Review of Depression Prevention Programs for Children and Adolescents: Factors That Predict Magnitude of Intervention Effects. Journal of Consulting and Clinical Psychology, 77(3), 486-503. https://doi.org/10.1037/a0015168

Sun, Y., Fu, Z., Bo, Q., Mao, Z., Ma, X., \& Wang, C. (2020). The reliability and validity of PHQ-9 in patients with major depressive disorder in psychiatric hospital. BMC Psychiatry, 20(1). https://doi.org/10.1186/s12888-020-02885-6

Waldinger, R. J., Vaillant, G. E., \& Orav, E. J. (2007). Childhood sibling relationships as a predictor of major depression in adulthood: A 30-year prospective study. American Journal of Psychiatry, 164(6), 949-954. https://doi.org/10.1176/ajp.2007.164.6.949

Weissman, M. M., Orvaschel, H., \& Padian, N. (1980). Children’s symptom and social functioning self-report scales comparison of mothers' and children's reports. Journal of Nervous and Mental Disease, 168(12), 736-740. https://doi.org/10.1097/00005053198012000-00005 
Table 1. Sample characteristics.

\begin{tabular}{lc}
\hline Characteristic & Frequency (\%) or Mean (SD) \\
\hline Female sex & $46(54.1 \%)$ \\
Age & $13.21(3.47)$ \\
Baseline (i.e., adolescence) & $30.86(3.63)$ \\
First follow-up & $34.97(4.05)$ \\
Second follow-up & $42.73(3.87)$ \\
Third follow-up & $46.64(4.09)$ \\
Fourth follow-up & $49.03(4.11)$ \\
Fifth follow-up & $44(51.8 \%)$ \\
Lifetime history of MDD & $16(18.8 \%)$ \\
MDD onset before baseline & $28(32.9 \%)$ \\
MDD onset after baseline & $50(58.8 \%)$ \\
Grandparental history of MDD & \\
Characteristics Measured in Adolescence & $15.17(9.28)$ \\
Depression & \\
Self-report & $1.28(0.22)$ \\
Peer dysfunction & $1.37(0.40)$ \\
Relations with siblings & $1.33(0.33)$ \\
Relations with parents & \\
Parent-report & $1.26(0.31)$ \\
Peer dysfunction & $1.22(0.26)$ \\
Dysfunction in sibling relations & $1.33(0.38)$ \\
Dysfunction in parent relations &
\end{tabular}

MDD = Major Depressive Disorder as assessed at each wave using the Schedule for Affective Disorders and Schizophrenia (Mannuzza et al., 1986) or equivalent for minors. Depression in adolescence was measured using the Center for Epidemiological Studies Depression Scale for Children. 
Table 2. Unstandardized coefficients (SEs) of models using self-rated adolescent peer dysfunction to predict adult depression.

Model

\begin{tabular}{|c|c|c|c|c|}
\hline Predictors & Unadjusted & Covariates $^{a}$ & Sibling Comparison & $\begin{array}{c}\text { Sibling Comparison }+ \\
\text { Covariates }^{\text {a }}\end{array}$ \\
\hline (Intercept) & $\begin{array}{c}-1.67 * * * \\
(0.43)\end{array}$ & $\begin{array}{l}-2.22 * \\
(0.85)\end{array}$ & $\begin{array}{l}-0.07 \\
(0.10)\end{array}$ & $\begin{array}{l}-0.51 \\
(0.55)\end{array}$ \\
\hline $\begin{array}{l}\text { Adolescent peer dysfunction } \\
\text { (self-reported) }\end{array}$ & $\begin{array}{c}1.28 * * * \\
(0.32)\end{array}$ & $\begin{array}{l}1.34 * * \\
(0.43)\end{array}$ & $\begin{array}{l}1.15^{* *} \\
(0.38)\end{array}$ & $\begin{array}{l}1.06^{*} \\
(0.48)\end{array}$ \\
\hline \multicolumn{5}{|c|}{ Covariates Measured in Adolescence } \\
\hline Sex [Female] & - & $\begin{array}{l}0.22 \\
(0.17)\end{array}$ & - & $\begin{array}{l}0.08 \\
(0.17)\end{array}$ \\
\hline Age & - & $\begin{array}{l}0.00 \\
(0.03)\end{array}$ & - & $\begin{array}{l}-0.01 \\
(0.03)\end{array}$ \\
\hline Baseline Depression & - & $\begin{array}{l}0.02 \\
(0.01)\end{array}$ & - & $\begin{array}{c}0.01 \\
(0.01)\end{array}$ \\
\hline $\begin{array}{l}\text { Dysfunction in sibling } \\
\text { relations (self-reported) }\end{array}$ & - & $\begin{array}{l}0.06 \\
(0.24)\end{array}$ & - & $\begin{array}{c}0.21 \\
(0.24)\end{array}$ \\
\hline $\begin{array}{l}\text { Dysfunction in parent } \\
\text { relations (self-reported) }\end{array}$ & - & $\begin{array}{l}0.02 \\
(0.27)\end{array}$ & - & $\begin{array}{c}0.03 \\
(0.29)\end{array}$ \\
\hline
\end{tabular}

$* p<.05 * * p<.01 * * * p<.001 .{ }^{\text {a }}$ Covariates were individual-level characteristics measured in adolescence. 
Table 3. Unstandardized coefficients (SEs) from models using parent-rated adolescent peer dysfunction to predict adult depression.

$\underline{\text { Model }}$

\begin{tabular}{|c|c|c|c|c|}
\hline Predictors & Unadjusted & Covariates $^{a}$ & Sibling Comparison & $\begin{array}{c}\text { Sibling Comparison }+ \\
\text { Covariates }^{\text {a }}\end{array}$ \\
\hline (Intercept) & $\begin{array}{l}-0.73 * \\
(0.33)\end{array}$ & $\begin{array}{l}-0.64 \\
(0.62)\end{array}$ & $\begin{array}{l}-0.08 \\
(0.10)\end{array}$ & $\begin{array}{l}-0.49 \\
(0.59)\end{array}$ \\
\hline $\begin{array}{l}\text { Adolescent peer dysfunction } \\
\text { (parent-reported) }\end{array}$ & $\begin{array}{l}0.56^{*} \\
(0.25)\end{array}$ & $\begin{array}{l}0.29 \\
(0.36)\end{array}$ & $\begin{array}{l}0.41 \\
(0.30)\end{array}$ & $\begin{array}{l}0.13 \\
(0.39)\end{array}$ \\
\hline \multicolumn{5}{|c|}{ Covariates Measured in Adolescence } \\
\hline Sex [Female] & - & $\begin{array}{l}0.13 \\
(0.19)\end{array}$ & - & $\begin{array}{l}0.04 \\
(0.18)\end{array}$ \\
\hline Age & - & $\begin{array}{l}-0.04 \\
(0.03)\end{array}$ & - & $\begin{array}{l}-0.03 \\
(0.03)\end{array}$ \\
\hline Baseline Depression & - & $\begin{array}{l}0.02 * \\
(0.01)\end{array}$ & - & $\begin{array}{l}0.01 \\
(0.01)\end{array}$ \\
\hline $\begin{array}{l}\text { Dysfunction in sibling } \\
\text { relations (parent-reported) }\end{array}$ & - & $\begin{array}{l}0.18 \\
(0.48)\end{array}$ & - & $\begin{array}{l}0.41 \\
(0.41)\end{array}$ \\
\hline $\begin{array}{l}\text { Dysfunction in parent } \\
\text { relations (parent-reported) }\end{array}$ & - & $\begin{array}{l}0.10 \\
(0.28)\end{array}$ & - & $\begin{array}{l}0.07 \\
(0.27)\end{array}$ \\
\hline
\end{tabular}

$* * * p<.001 . * * p<.01 . *{ }^{*} p<.05 .{ }^{\text {a }}$ Covariates were individual-level characteristics measured in adolescence. 
2. Dysfunction in sibling relations (self-reported)

3. Dysfunction in parent relations (self-reported)

4. Peer Dysfunction (parent-reported)

5. Dysfunction in sibling relations (parent-reported)

6. Dysfunction in parent relations (parent-reported)

7. Depressive Symptoms

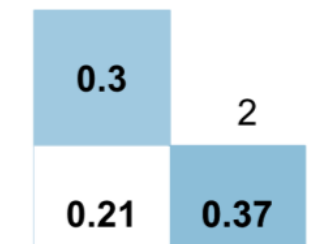

$\begin{array}{llll}0.37 & 3\end{array}$

\begin{tabular}{l|l|l}
0.51 & 0.38 & 0.17
\end{tabular}

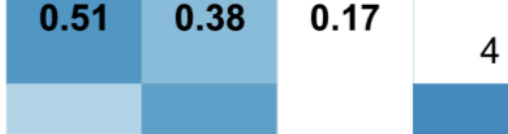

\begin{tabular}{l|l|l|l}
0.26 & 0.48 & 0.19 & 0.55
\end{tabular}

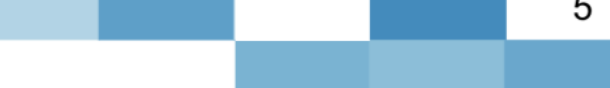

\begin{tabular}{|l|l|l|l|l|}
\hline 0.05 & 0.2 & 0.42 & 0.36 & 0.46 \\
\hline
\end{tabular}

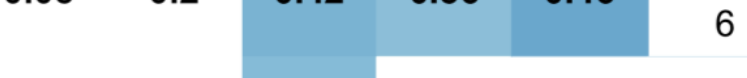

\begin{tabular}{|l|l|l|l|l|l}
0.12 & 0.09 & 0.39 & 0.01 & 0.14 & 0.13
\end{tabular}
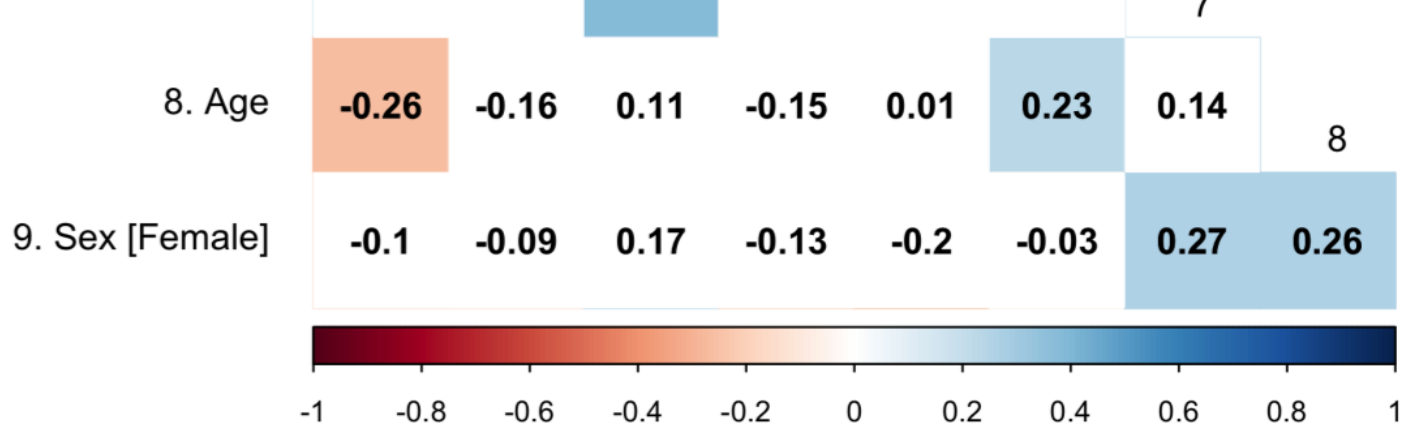

Figure 1. Correlations between individual characteristics in adolescence. All values reflect Pearson correlations, except that correlations involving sex are point-biserial correlations. Significant correlations $(p<.05)$ are shaded. The color of the shading reflects the direction of the correlation (red $=$ negative, blue $=$ positive) and the degree of shading represents the strength of the correlation. 


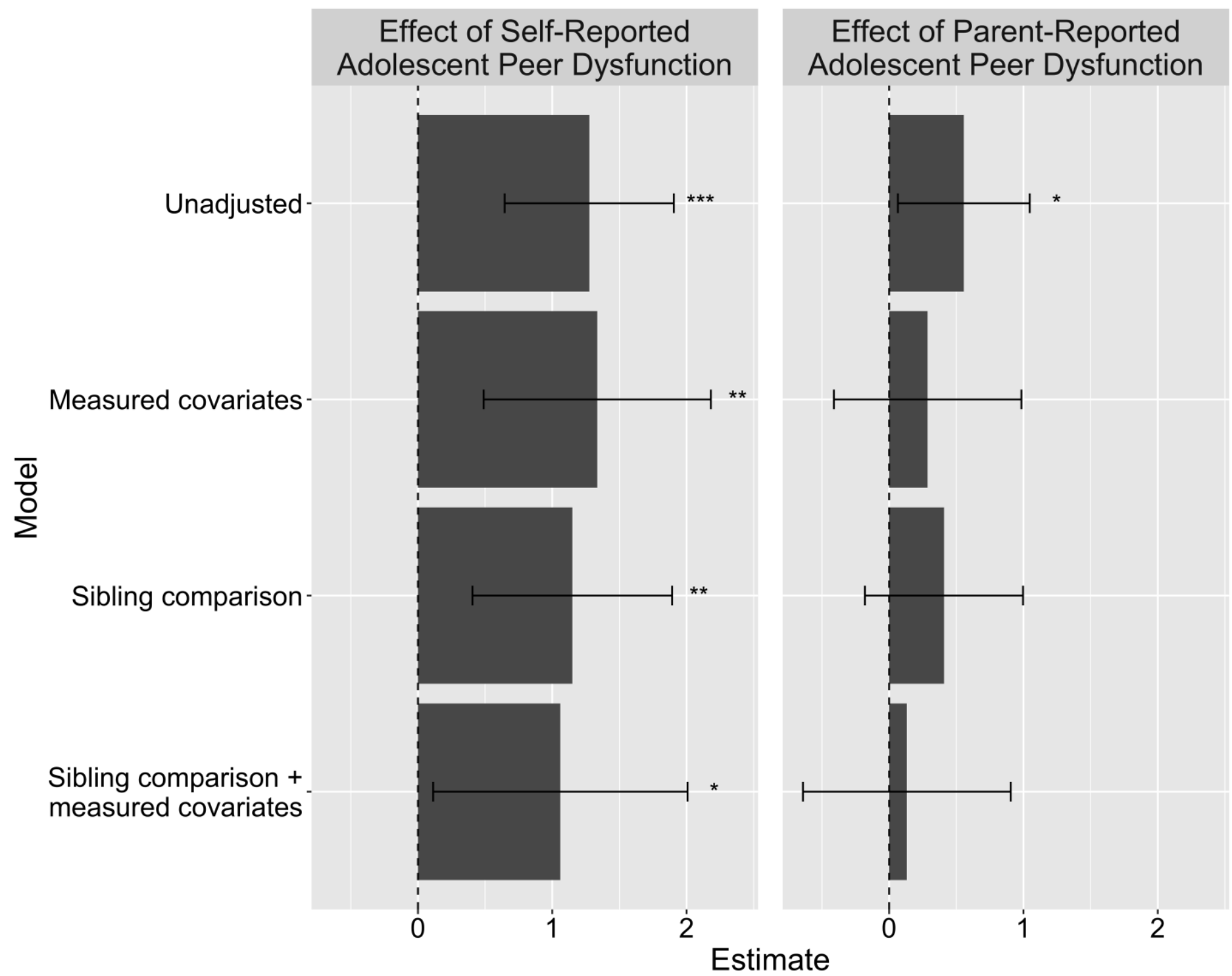

Figure 2. Unstandardized associations between self-reported (left) and parent-reported (right) adolescent peer dysfunction and adult depression. Error bars represent $95 \%$ confidence intervals. $* p<.05 * * p<.01 * * * p<.001$ 